\title{
Goals for Reaching Optimal Wellness (GROWell): A Clinical Trial Protocol of a Digital Dietary Intervention for Pregnant and Postpartum Women with Prenatal Overweight or Obesity
}

\section{Leigh Ann Simmons ( $\square$ lasimm@ucdavis.edu )}

University of California Davis https://orcid.org/0000-0001-9400-5723

Jennifer Phipps

University of California Davis https://orcid.org/0000-0003-0004-2908

Courtney Overstreet

University of California Davis

Elizabeth Bechard

University of California Davis

Siwei Liu

University of California Davis

Cheryl Walker

University of California Davis

Devon Noonan

University of California Davis

\section{Study protocol}

Keywords: Gestational weight gain, Postpartum weight retention, Mobile health intervention, Overweight, Obesity, Pregnancy

Posted Date: July 19th, 2021

DOI: https://doi.org/10.21203/rs.3.rs-397736/v1

License: (9) This work is licensed under a Creative Commons Attribution 4.0 International License.

Read Full License 


\section{Abstract}

Background: Excess gestational weight gain (GWG) is associated with many pregnancy complications and health risks for mothers and infants. Likewise, postpartum weight retention (PPWR) has long-term health consequences for women. Excess GWG among women who enter pregnancy with overweight or obesity have worse obstetric outcomes and increased PPWR compared to women who gain within Institute of Medicine guidelines.

Methods: This study is a blinded, randomized clinical trial of GROWell: Goals for Reaching Optimal Wellness, a mHealth tool designed to improve diet quality among women who enter pregnancy with overweight or obese BMIs in order to help them achieve appropriate GWG and safe postpartum pregnancy weight loss. Overweight and obese (BMI B25 and <40) women with singleton, uncomplicated pregnancies will be recruited and randomly assigned to an attention control or intervention arm. The intervention group will receive goal-oriented text messages regarding dietary choices, while the attention control group will receive text messages about healthy pregnancy, labor, delivery and early infancy. Both groups will complete online surveys at baseline (26-28 weeks), follow up (36-38 weeks), 3 months postpartum, and 6 months postpartum.

Discussion: This study protocol describes the details of the GROWell randomized clinical trial. The results of this trial will provide data to support the use of an evidence-based mHealth tool to be integrated into clinical practice to reduce excess GWG and PPWR among pregnant women with overweight and obese BMls, a resource that is currently lacking.

Trial registration: ClinicalTrials.gov identifier: NCT04449432. Registered on June 26, 2020.

\section{Adminstrative Information}




\begin{tabular}{|c|c|}
\hline Title $\{1\}$ & $\begin{array}{l}\text { Goals for Reaching Optimal Wellness ( GROWell): A Clinical Trial Protocol of a } \\
\text { Digital Dietary Intervention for Pregnant and Postpartum Women with Prenatal } \\
\text { Overweight or Obesity }\end{array}$ \\
\hline $\begin{array}{l}\text { Trial registration }\{2 \mathrm{a} \\
\text { and } 2 \mathrm{~b}\} \text {. }\end{array}$ & ClinicalTrials.gov identifier: NCT04449432. Registered on June 26, 2020 \\
\hline Protocol version (1) & October 15, 2020. Version 1. \\
\hline Funding $\{4\}$ & $\begin{array}{l}\text { This study is funded by the National Institutes of Health, National Institute of } \\
\text { Nursing Research, R01NR017659. }\end{array}$ \\
\hline Author details $\{5 \mathrm{a}\}$ & $\begin{array}{l}\text { Leigh Ann Simmons, Ph.D., M.F.T. }{ }^{*}{ }^{*} \text {, Jennifer E. Phipps, Ph.D. }{ }^{1} \text {, Courtney } \\
\text { Overstreet, CCRP2 }{ }^{2} \text { Elizabeth Bechard, B.A. }{ }^{3} \text {, Siwei Liu, Ph.D. }{ }^{1} \text {, Cheryl Walker, } \\
\text { M.D. }^{2} \text {, Devon Noonan, Ph.D., MPH, FNP-BC } \\
{ }^{1} \text { University of California Davis, Human Ecology Department, Davis, CA } 95616 \\
{ }^{2} \text { University of California Davis Health, Obstetrics and Gynecology Department, } \\
\text { Sacramento, CA } 95817 \\
{ }^{3} \text { Duke Integrative Medicine, Durham, NC } 27705 \\
{ }_{\text {Duke University School of Nursing, Durham, NC } 27710} \\
{ }^{*} \text { corresponding author }\end{array}$ \\
\hline $\begin{array}{l}\text { Name and contact } \\
\text { information for the } \\
\text { trial sponsor }\{5 b\}\end{array}$ & N/A \\
\hline Role of sponsor $\{5 c\}$ & $\mathrm{N} / \mathrm{A}$ \\
\hline
\end{tabular}

\section{Introduction}

\section{Background and rationale $\{6 a\}$}

Excess gestational weight gain (GWG), defined as gaining more weight during pregnancy than Institute of Medicine (IOM) guidelines for prepregnancy body mass index (BMI), is a significant public health issue, especially for women who enter pregnancy with overweight or obese BMls. National data show that more than half of women with $\mathrm{BMI} \geq 25$ gain excess gestational weight with one study demonstrating that 
$64.1 \%$ of overweight women and $58.2 \%$ of obese women gain excessive gestational weight.(2) Compared to women who gain within IOM guidelines, excess GWG among women with prenatal overweight or obesity is associated with increased risk for poor obstetric, neonatal, and maternal outcomes,(3-14) including gestational diabetes, preeclampsia, cesarean delivery, preterm birth, fetal growth abnormalities, and postpartum weight retention (PPWR) at 6 months post-birth, a time point now recognized as critical for predicting whether women will ever return to their prepregnancy weight.(15-17) Women with excess GWG also are more likely to never return to prepregnancy weight and to move to the next BMI class (e.g., normal weight prepregnancy to overweight post-pregnancy), thereby increasing the risk status of any subsequent pregnancies.(13, 18-21) Lastly, excess GWG has long-term repercussions. Women with excess GWG have increased risk for cardiometabolic disease into mid- and later life, while their infants have increased risk for overweight, obesity, cardiometabolic disease, poor cognition, and mood disorders from childhood through adulthood. $(9,11,22-26)$

Attempts to prevent excess GWG have had limited impact on the problem, particularly in overweight and obese women.(27) Over the last decade, the average proportion of women gaining gestational weight within IOM recommendations has slightly decreased, while the mean GWG has slightly increased.(28) These trends may be due in part to the fact that interventions to reduce GWG have varied widely in approaches and success rates, including few large randomized trials. Interventions have been delivered by dieticians, health coaches, midwives, and health educators. $(29,30)$ Individual-based interventions have included both in-person and telephonic sessions and ranged in intensity from four 30-60 minute sessions $(31,32)$ to one 5-minute session after every prenatal visit.(33) Group-based interventions have been delivered primarily in person and ranged in intensity from a low of three 1-hour sessions(34) to 16 90-minute sessions.(35) Results from these trials have shown that higher intensity interventions that provided at least some individualized support were associated with lower GWG compared to control conditions. However, even some high intensity interventions had null results. Poston et al. investigated a lifestyle intervention that consisted of eight 90-minute group sessions providing general diet and physical activity information and found no improvements over usual care.(36)

Previously developed interventions have included technology but found mixed results, despite the fact that technology: (1) extends reach; (2) is easily tailored to meet individual needs; and (3) provides the frequency of contact necessary for sustained behavioral changes with minimal human, financial, and time resources. $(37,38)$ Interventions that have employed technology have varied widely in approach and outcomes. MomTech participants who received two daily text messages and four lifestyle appointments with a midwife had lower GWG than control participants, but the study was not powered for statistical significance.(39) e-Moms Roc included an informational website with mobile phone applications, weight gain trackers, and goal-setting tools for diet and physical activity. Only women with access to the personalized weight, diet, and physical activity tools had lower GWG.(40) The VideoDoctor trial found no improvement in GWG from an actor-portrayed doctor delivering two brief video messages about diet, exercise, and weight gain.(41) These findings reflect the conclusion of a recent systematic review: "... while technology has potential as a safe and therapeutic tool, further RCTs examining its effectiveness... 
are required...particularly among overweight and obese pregnant women" (p. 760).(42) Moreover, none of these studies continued into the postpartum period, which may be critical for limiting PPWR.

\section{Objectives $\{7\}$}

We propose to conduct one of the first, fully powered RCTs to test the efficacy of GROWell, a personalized digital dietary intervention for women who entered pregnancy with an overweight or obese BMI. Our study is based on the premise that a theoretically driven, standalone mHealth tool with a high intensity intervention, timed during pregnancy through six months postpartum, will help women with overweight and obesity to gain appropriate gestational weight and prevent PPWR. GROWell employs algorithms and text messaging to personalize food preferences, goal-setting, diet tracking, skills training, and adherence feedback to promote dietary change, thereby using limited human resources. GROWell is based on Selfregulation Theory,(43-46) which posits that individuals must consciously plan their actions and monitor their behaviors to achieve desired goals (Figure 1). Previous studies based on Self-regulation Theory(47) show that goal attainment is enhanced when individuals receive support, including problem-solving skills, that can be applied to their actions.

\section{Trial design $\{8\}$}

We will conduct a blinded RCT of GROWel/ to determine whether the intervention is efficacious at achieving GWG within IOM-recommended ranges and reducing six-month PPWR compared to an attention control. Figure 2 provides an overview of the study design. Due to the emergence of the COVID19 pandemic, we have modified our study to allow for the protocol to be implemented remotely. Participants can have the option to consent and complete surveys in the clinic with study staff, or remotely in their own homes.

\section{Methods}

\section{Participants, interventions and outcomes}

\section{Study setting $\{9\}$}

We will recruit subjects from University of California Davis Obstetrics and Gynecology clinics and the community around UC Davis. Counties included in our catchment area include: Yolo, Sacramento, Solano, Napa, Sonoma, San Joaquin, Alameda, Stanislaus, Santa Clara, San Mateo, Santa Cruz, San Francisco and Marin.

\section{Eligibility criteria $\{10\}$}

We will recruit women aged 18-44 who are 10-16 weeks gestation of a confirmed pregnancy (i.e., ultrasound/heartbeat detected) and nulliparous or $>12$ months since previous birth. Women with $\mathrm{BMI} \geq 25$ and $<40$ who are willing to receive and respond to texts using their own cell phone or a study-provided phone will be included. Exclusion criteria include: (a) known pregnancy or fetal complications/high-risk 
status (e.g., fetal chromosomal abnormality, chronic hypertension), (b) multiple gestation, and (c) unable to read/write English. We also will exclude women $\geq 45$ years and at the extreme high end of the BMI range $(\geq 40)$ due to increased risk for pregnancy complications, and thus higher likelihood of dropping out. $(48,49)$ Smokers and women who quit smoking $<6$ months prior to recruitment will be excluded given data that show smoking is associated with increased risk for pregnancy complications $(50,51)$ and smoking cessation affects eating patterns and weight.(52-54) Women with untreated depression (i.e., not on a stable class/dose of antidepressant for $\geq 6$ months or with Edinburgh Scores $>12$ based on first trimester clinic screening) will be excluded due to documented relationships between depression, appetite changes, and eating behaviors. $(55,56)$

\section{Who will take informed consent? $\{26 a\}$}

Informed consent will be provided by participants online through DocuSign either at home on their personal devices or at their UC Davis clinic on a study-provided device via a clinical research coordinator.

\section{Additional consent provisions for collection and use of participant data and biological specimens $\{26 \mathrm{~b}\}$}

N/A

\section{Interventions}

\section{Explanation for the choice of comparators $\{6 b\}$}

Research on behavioral trials has suggested that significant differences between intervention and usual care or non-attention controls may contribute to inflated results or lack of clarity regarding whether effects are the result of actual intervention components or mere attention.(57-59) In our preliminary work, we developed an attention control delivered using text messaging to reduce the potential placebo effect that interacting with our mHealth system may have on pregnancy weight gain and postpartum weight loss. By providing information to control participants that is specific to pregnancy, labor, delivery, and early infancy, but not to diet, we will isolate the effects of diet-focused goal-setting, self-monitoring, and feedback in GROWell as compared to receipt of a general pregnancy education intervention. Based on information we collect about partner and employment status, parity, breastfeeding intentions, etc., participants assigned to the attention control will receive: (a) pregnancy, fetal development, labor and delivery, and early infancy educational messages provided via text once weekly between enrollment and 37 weeks gestation; and (b) education on infant development via weekly texts from birth to 6 months postpartum (see Table 1).

Table 1. Sample text messages 


\begin{tabular}{|c|c|c|}
\hline \multirow{2}{*}{\multicolumn{3}{|c|}{ Characteristics }} \\
\hline & & \\
\hline All & $\begin{array}{l}\text { Eating iron-rich foods, such as } \\
\text { [examples specific to dietary } \\
\text { preferences] supports your } \\
\text { baby's development. }\end{array}$ & $\begin{array}{l}\text { Trouble sleeping? Follow these tips for better } \\
\text { ZZZZs. https://cle.clinic/2wCSRzM }\end{array}$ \\
\hline $\begin{array}{l}\text { Working } \\
\text { outside the } \\
\text { home }\end{array}$ & $\begin{array}{l}\text { Pack a healthy lunch instead of } \\
\text { eating out to lower sodium and } \\
\text { sugar intake. }\end{array}$ & $\begin{array}{l}\text { Time to find out what kind of leave you have at your work. Talk } \\
\text { to human resources and make a plan. }\end{array}$ \\
\hline Has partner & $\begin{array}{l}\text { Plan this week's meals with } \\
\text { your partner. Pick } 3 \text { recipes } \\
\text { from this list to increase intake } \\
\text { of lean proteins. }\end{array}$ & $\begin{array}{l}\text { Adding a new baby to the family can affect your relationship. } \\
\text { Take at least } 1 \text { hour each week to spend alone time together. }\end{array}$ \\
\hline \multicolumn{3}{|r|}{ Postpartum } \\
\hline Breastfeeding & $\begin{array}{l}\text { Getting enough calcium is } \\
\text { important while breastfeeding. } \\
\text { 1oz of almonds is a great snack } \\
\text { that provides healthy fats, } \\
\text { calcium, and fiber. }\end{array}$ & $\begin{array}{l}\text { Eye contact with baby is important for communication. Look } \\
\text { into your baby's eyes while feeding [him/her] rather than } \\
\text { watching TV or working on your cell phone. }\end{array}$ \\
\hline $\begin{array}{l}\text { Returning to } \\
\text { work outside } \\
\text { the home }\end{array}$ & $\begin{array}{l}\text { Prepare mason jar salads on } \\
\text { Sunday to make sure you keep } \\
\text { eating dark, leafy greens when } \\
\text { you go back to } \\
\text { work. http://bit.ly/3bXbSwX }\end{array}$ & $\begin{array}{l}\text { Returning to work is hard enough, but it's really hard when } \\
\text { baby cries when you leave [him/her] with a caregiver. Know } \\
\text { that baby is learning to trust other adults and that mom comes } \\
\text { back when she leaves. Both are important for baby to develop } \\
\text { secure attachment. }\end{array}$ \\
\hline
\end{tabular}

\section{Intervention description $\{11$ a $\}$}

Upon enrollment, participants take a brief survey on their specific dietary behaviors, their readiness and self-efficacy to change these behaviors, and dietary preferences/restrictions. The results are tallied, and our prescription algorithm assigns two, personally tailored dietary change goals. The algorithm prioritizes goals in highest need of change and for which the participant has high self-efficacy and readiness. Figure 3 demonstrates an example of the mobile health platform with sample messaging. Participants engage with GROWel/Monday through Friday. Once weekly, participants report via text or the application how many days in the previous week they adhered to their goals. The remaining four days, participants receive supportive, goal-focused text messages with problem-solving skills and dietary tips tailored to the previous week's progress on their goal. When participants do not respond, we use a complex text-back protocol that has produced weekly self-monitoring adherence rates between $72 \%$ and $84 \%$ at 12 months. $(60,61)$

During the prenatal period, the library of dietary behavioral goals is consistent with controlling energy intake and meeting nutrition recommendations for pregnancy. These recommendations include increasing micronutrient needs such as iron, calcium, and folic acid, consuming a balanced diet of whole grains, healthy fats, dietary fiber, fruits, vegetables, and lean protein, and increasing caloric intake by 300 
calories daily after 14 weeks. As women successfully meet their goals with $>70 \%$ adherence (i.e., at least 5 out of 7 days) for 2 consecutive weeks, they will be assigned the next goal for which they have the highest need combined with the highest readiness to change.

Approximately 10-14 days post-delivery, participants will retake the GROWell dietary assessment online. This will collect information including breastfeeding status (i.e., full, partial, none), and the prescription algorithm will assign two new, personally tailored dietary change goals based on breastfeeding status to meet micronutrient needs (e.g., calcium, iron, protein, etc.) and promote safe postpartum weight loss (1/2-2 pounds per week). Among breastfeeding mothers, continued breastfeeding will be reassessed with weekly self-monitoring texts. When breastfeeding status changes (i.e., full or partial to partial or none), the participant will receive a link to retake the GROWell assessment online so that new dietary goals can be prescribed that reflect non-breastfeeding status. See Table 1 for sample texts.

\section{Criteria for discontinuing or modifying allocated interventions $\{11 \mathrm{~b}\}$}

Physical risks associated with pregnancy include pregnancy loss, diagnosis of a fetal anomaly, complications of pregnancy that necessitate admission to the antepartum unit, preterm delivery, and stillbirth. Women in either the attention control or intervention arms who experience any of these complications will be discontinued from the study and be categorized as experiencing pregnancy complications in the study database so that rates of these complications can be compared between the two arms. All of these events will be reported as adverse events or serious adverse events according to severity and likelihood that the event was study-related. If subjects withdraw from the study voluntarily, their data will be removed from the database.

\section{Strategies to improve adherence to interventions $\{11 \mathrm{c}\}$}

We will measure adherence to text-based self-monitoring cumulatively by week as the number of times a participant responds to weekly prompts to report on her progress to the number of times she was prompted, calculated to a percent. Based on previous trials, we will consider good cumulative adherence to be $\mathrm{a} \geq 70 \%$. Rates will be calculated separately for the prenatal and postpartum periods. We will measure adherence to prescribed goals cumulatively by week as the proportion of goals for which a participant has "good adherence" during the previous week. Because goals change over time with improved adherence, participants are working on 2 goals at once, and some behaviors are easier to change than others, we consider average weekly adherence to be good at $\geq 50 \%$. Rates will be calculated separately for the prenatal and postpartum periods.

To determine whether adherence to GROWell affected our primary outcomes, we will use latent growth curve models with data from GROWell participants only to examine whether there are systematic trends in prenatal self-monitoring adherence rates and prenatal goal adherence rates. If systematic trends are found, we will examine whether latent factors representing changes in adherence over time (e.g., intercept, slope) predict GWG and PPWR in the structural equation modeling (SEM) framework. If no systematic trend is found, we will compute individual-specific summary statistics (e.g., mean and variance) of the 
adherence measures and test them as predictors of GWG and PPWR. The first model will control only for BMI groups. The second model will include baseline demographic and health behavior variables (race/ethnicity, age, parity, diet quality, physical activity, breastfeeding [postpartum model], and GWG [postpartum model]). The final model will add the recruitment source.

\section{Relevant concomitant care permitted or prohibited during the trial $\{11 \mathrm{~d}\}$}

Trial participants are expected to receive routine clinical care for their pregnancy separate from this study. They are prohibited from participating in other trials.

\section{Provisions for post-trial care $\{30\}$}

$\mathrm{N} / \mathrm{A}$

\section{Outcomes $\{12\}$}

Primary Outcomes: Excess GWG and PPWR. GWG will be calculated as: [weight (delivery) $^{-}$ weight (preconception)]. Women will be categorized as gaining excess gestational weight if they started pregnancy as overweight and gained more than 25 pounds or started pregnancy as obese and gained more than 20 pounds. PPWR will be calculated as: [weight(6 months postpartum) - weight (preconception)]. Women will be categorized as experiencing PPWR if postpartum weight at 6 months is greater than $1.05^{\star}$ preconception weight. We have selected the 6-month time point given research that shows if a woman is not within $5 \%$ of her prepregnancy weight by 6 months postpartum, she is significantly less likely to return to prepregnancy weight. $(13,15,16)$ We will compare the proportions of women in each group who gain excess gestational weight and retain postpartum weight versus examining statistical differences in weights between because: (1) previous intervention studies aimed at reducing GWG have demonstrated that, while reductions in weight gain are achieved, women are still gaining more than IOM recommendations for prepregnancy BMl;(30) (2) in clinical obstetric practice, the current guidelines still use the classifications for gestational weight gain ("inadequate," "appropriate," and "excessive") as a guide for weight/diet counseling in postpartum and interpregnancy care;(62) and (3) raw weight gains do not have ecological validity when comparing across different starting weights (e.g., a $150 \mathrm{lb}$ woman and a $200 \mathrm{lb}$ woman may both gain $20 \mathrm{lbs}$ but it is a different percent of starting weight and may or may not reflect a clinically meaningful difference in appropriate weight gain).

Exploratory Maternal, Obstetric, and Neonatal Outcomes. Although we are not powered to detect significant effect size differences in maternal, obstetric, and neonatal outcomes, robust evidence suggests excess GWG negatively affects these outcomes. $(3,6,7,19,63,64)$ We may obtain important preliminary data for future pragmatic trials of GROWel/ where we are powered to examine differences in one or more of these factors. Blinded research staff will abstract the following data from the EHR: (a) delivery type (cesarean or vaginal), and (b) fetal growth abnormalities (small-for-gestational age, largefor-gestational-age, macrosomia) or none. Pregnancy complications (e.g., gestational diabetes, hypertension, preeclampsia, eclampsia, placental abruption, fetal death, antepartum admission, preterm 
birth with and without NICU admission) will be documented categorically using a combination of participant self-report, the 26-28 week and 36-38 week prenatal online assessments, and the EHR. Rates of each of these outcomes will be calculated as a percent (the total number of events/total number of participants) for each arm.

\section{Participant timeline $\{13\}$}

Baseline. Prior to treatment assignment, each participant will complete an online baseline survey. All participants will be paid $\$ 45$ for completion of this survey, which will take $\sim 45$ minutes. The survey will include basic demographics, parity, diet quality, depression/anxiety, and physical activity. A Bluetooth scale will be mailed to each participant, who will receive written and video instructions on how to link the scale with their phone and how to provide weights at the same time points as the online assessments. See more information on weight calculations below.

Online Follow-ups. Each online follow-up will remind participants to weigh themselves with their Bluetooth scale. For the postpartum period, breastfeeding status will be assessed weekly until cessation. At each time point, the following information will be assessed:

1. 26-28 weeks gestation: diet quality, new diagnoses of pregnancy complications (e.g., gestational diabetes, hypertension, etc.). Time required: 30 minutes, compensation: \$15

2. 36-38 weeks gestation: diet quality, depression/anxiety, physical activity, demographics update, new diagnoses of pregnancy complications (e.g., gestational diabetes, hypertension, etc.). Time required: $\sim 45$ minutes, compensation: $\$ 60$

3. 3 months postpartum: diet quality. Time required: 30 minutes, compensation: $\$ 15$

4. 6 months postpartum: diet quality, depression/anxiety, physical activity, demographics update, and intervention satisfaction. Time required, $\sim 45$ minutes, compensation: $\$ 90$

\section{Sample size $\{14\}$}

Our team's preliminary work showed the proportion of women who gained excess gestational weight was $37 \%$ in the group using mHealth vs. $66 \%$ receiving usual care, a 29 -unit difference. ${ }^{82}$ Using a text messaging plus in-person education intervention among obese pregnant women, Soltani et al. ${ }^{58}$ found that $28 \%$ in the intervention group gained excess gestational weight vs. $50 \%$ in the control group, a $22-$ unit difference. Based on power calculations for fitting a standard logistic regression including all covariates, the proposed analyses for aims 1 and 2 have $80 \%$ power to detect a minimal adjusted difference of 15 percentage units (OR: 0.55) between the intervention arms for excess gestational weight gain and postpartum weight retention with a total sample size of 354 for a 2-sided significance level of 0.05. This effect size is consistent with previous studies and is nearly representative of a Cohen's small effect, 10 percentage units (OR: 0.67). Power calculations were performed using PROC POWER in SAS version 9.4. To account for drop-out of participants, we will recruit an extra 126 participants for a total of 480 women for this study; 240 will be randomized to GROWell and 240 to the Attention Control. 


\section{Recruitment \{15\}}

Using the automated appointment system in the electronic health record, clinical research coordinators (CRCs) will generate weekly lists of patients receiving prenatal care at one of eight obstetric clinics affiliated with an academic medical center who meet preliminary eligibility criteria (i.e., age, gestational week, singleton pregnancy, parity, BMI). CRCs will either: (1) approach potential participants at their usual obstetric visit after introduction from a member of their provider team to describe the study and receive verbal consent to administer formal prescreening; or (2) send opt-out letters from the clinic director and follow up via phone with potential participants who do not opt out to determine interest in study participation and conduct screening. We also will post flyers in the community and use a targeted social media campaign on Facebook and Instagram. All women who agree to participate will consent using an IRB-approved digital form, which can be administered virtually or in person on a mobile device.

\section{Assignment of interventions: allocation}

\section{Sequence generation $\{16 \mathrm{a}\}$}

Treatment assignment will occur after completion of the baseline computer assisted interview. Computerized stratified randomization with permuted blocks of size 3 will be used to assign eligible participants to GROWell $(n=240)$ or the attention control $(n=240)$. Prepregnancy BMI categories (overweight=BMI 25-<30; class 1 obese=BMI 30-<35; class 2 obese=BMI $35<40$ ), race, and recruitment method (clinic or via social media) will be the stratification variables.

\section{Concealment mechanism $\{16 \mathrm{~b}\}$}

The software platform used to deliver the treatments (intervention or attention control) will blind the treatment assignments from research staff.

\section{Implementation \{16c\}}

Participants will be enrolled online or blinded clinical research coordinators will enter their information into the online system to enroll participants. The enrollment process is linked to the software platform used to deliver the treatments, which will then randomize participants into the intervention or attention control groups.

\section{Assignment of interventions: Blinding}

\section{Who will be blinded $\{17 a\}$}

Research staff, study participants, and care providers will be blinded to the intervention assignments. The assignment of the intervention occurs in the software platform used in this study, and the intervention group is only visible from the software engineer's view of the software. 
The study statistician will remain blinded as well as the rest of the research staff (including clinical research coordinators and care providers). Upon reviewing the study statistician's reports, if the Data Safety and Monitoring Board (DSMB) believes there is any reason to unblind the data, the data may be unblinded for the DSMB (everyone else will remain blinded). The data to match each participant with their respective group can be provided by the software company that designed the platform used to deliver the treatments.

\section{Data collection and management}

\section{Plans for assessment and collection of outcomes $\{18 \mathrm{a}\}$}

We will use validated instruments and questions to describe the sample characteristics and assess key behavioral factors that may influence our primary outcomes.

Demographics: Race/ethnicity and clinic type are included as stratification variables. We will record age as a continuous variable. Categorical variables include: educational level ( $<12$ years, $12+$ years), partner status (yes, no), health insurance type (private, public), employment status (full-time, part-time, not employed), and parity (primagravida yes/no).

Diet Quality: Online assessments at 36-38 weeks and 6 months postpartum will capture diet using the web-based Automated Self-Administered 24-Hour Dietary Assessment Tool (ASA24®)(C).(65) Developed by the $\mathrm{NCl}$, the ASA24® enables automatically coded, self-administered 24-hour diet recalls. Participants report on foods they consumed in the previous day calculated from midnight-to-midnight, including form, how it was prepared, portion sizes, and when and where it was consumed. For both of these instruments, we will use Diet*Calc software to calculate an Alternate Healthy Eating Index-2015 (AHEI-2015) value for use in all analyses.(66-68) At each online assessment (baseline, 24-26 weeks, 36-38 weeks, 3 months and 6 months postpartum) we will also ask participants to fill out the Rapid Eating Assessment for Participants (REAP) questionnaire. The REAP survey is faster to complete than the ASA24, thus we ensure that even if participants do not complete the ASA24, we are obtaining diet quality information across the timeline each participant is enrolled in the study.

Physical Activity: We will assess physical activity using the Kaiser Physical Activity Survey (KPAS),(69) which has been validated for use in pregnant(70) and nonpregnant women,(69) making it a good instrument to assess physical activity across the prenatal and postpartum periods. Test-retest reliability is good at $r=.84$ and $r=.79-.91$ for pregnant and nonpregnant women respectively. The KPAS shows concordant validity ( $r=.71-.84)$ with the Pregnancy Physical Activity Questionnaire, a widely used instrument to assess prenatal physical activity.

Breastfeeding: We will measure breastfeeding categorically as exclusive, partial (some formula use and/or solid introduction), or none.

Depression/Anxiety: We will measure symptoms of depression and anxiety using the Edinburgh Postnatal Depression Scale (EPDS),(71-73) which has been validated for pregnant and postpartum 
women. Total scores have been validated as a measure of clinically significant depressive symptoms, while the anxiety subscale (items $3-5$ ) has been validated as a measure of clinically significant anxiety symptoms.(74)

\section{Plans to promote participant retention and complete follow-up $\{18 \mathrm{~b}\}$}

Our previous long-term trials had retention rates of $80-96 \%$ between 6 and 24 months in nonobstetric (60, 61) and obstetric (75-77) participants. We have implemented a number of strategies to promote retention, including graduated remuneration, virtual data collection, multiple contact numbers for the participant and known contacts, and quarterly data reviews. The intervention is delivered remotely to reduce participant burden. We also will recruit more participants than the minimum sample size needed to achieve statistical power. However, we will examine dropouts to determine whether there are any systematic differences between withdrawals from the groups. Should we find systematic differences, we will generate propensity scores matched on reasons for leaving and analyze all available data to determine any differences in outcomes using all data vs. only complete data.

\section{Data management $\{19\}$}

This study is conducted virtually, thus subjects will be submitting data through a web-based mobile app platform that is third-party certified under the HITRUST Common Security Framework. Although these servers are secured and password-protected, should any of the servers become compromised, there is a risk of loss of confidentiality. Additionally, Electronic Medical Record (EMR) data will be extracted from subject medical records and stored on secure servers on encrypted and password protected computerscoded to hide the identify of subjects. Participants will receive the electronic version of the HIPAA Authorization and Records Request forms for electronic signature with DocuSign to allow for EMR data to be accessed. There is a risk that the codes to link identifiable information could be exposed, but every possible strategy will be taken to prevent this. It is highly unlikely that an encrypted computer with a strong password will be compromised.

Our use of electronic systems for data entry allows us to continuously monitor data for acceptability. Our data analyst will monitor the data that are collected from participants. The software system we are using in this trial allows us to run a range of statistical procedures in real time on a rolling basis. The statistician will lead the review distribution of collected survey data monthly during the recruitment phase. We will evaluate the data for systematic errors, outlying values, and item completion rates. We will take corrective steps and adjust the protocol to remedy any systematic errors in data collection. We will also verify outlying values, as appropriate.

The mobile app platform used in this trial does not require client local software and can be accessed from anywhere on the Internet secured on a UC Davis server. This platform provides: (1) a streamlined process for rapidly building a database; (2) an intuitive interface for collecting data (with data validation and audit trail); (3) automated export procedures for seamless data downloads to common statistical 
packages (SAS, SPSS, etc.); (4) branching logic, file uploading, and calculated fields; and (5) a quick and easy protocol set-up.

Authentication to access the mobile app platform occurs through centralized secure pathways. All connections to the system, both external and internal, occur over encrypted channels. Access to components of the system is role-based and can only be granted by administrators of the system. All collected information is stored on a standalone database server. The database server resides behind the UC Davis Medical Center (UCDMC) internal firewall and access to the server is controlled via firewall rules. All collected data is backed up daily, both on the local server and by the UCDMC backup system.

\section{Confidentiality $\{27\}$}

We will use the standard operating procedures that have been used in our prior trials for survey conduct and data management. These procedures have been developed and used in many studies and we will adhere to these procedures for the proposed study. All paper files related to the study will be stored in a secure location at UC Davis in a locked file cabinet accessible only to key study personnel. The privacy, security, and durability of accumulated personal health information are of paramount importance. Our existing practices for collecting and storing electronic data meet or exceed the software industry's best practices for protecting personal data. Specifically: (a) physical access to computing resources (hard drives, servers, networking equipment) is secure, audited, and restricted to skilled personnel; (b) virtual access (login and system administration) is encrypted, audited, and limited to skilled personnel; (c) all databases are encrypted and access from the public Internet is precluded; (d) all software and servers are reachable only via a virtual private network (VPN) or multifactor authentication (MFA); and (e) all communication between our software's application programming interface (API) and third-party software is encrypted using SSL. Personnel must have access to perform regular maintenance duties. However, this constituency is limited to senior system administrators and the director of the research group.

Participant information will be entered into a secure research server, hosted and maintained in a remote data center. All software and servers are reachable only via logging in using multi-factor authentication. All communication between our software's application programming interface and third-party software is encrypted. Physical access to computing resources (hard drives, servers, networking equipment) is secure, audited, and restricted to skilled personnel; virtual access (login and system administration) is encrypted, audited, and limited to skilled personnel; all databases are encrypted and access from the public Internet is precluded. All software and servers are reachable only via a virtual private network (VPN) or multi-factor authentication; and all communication between our software's application programming interface and third-party software is encrypted using. Only research staff who have received CITI and HIPAA Certification and are listed on the UC Davis Institutional Review Board protocol will have access to the study database. Information in the database will not be shared with anyone outside the research team.

Plans for collection, laboratory evaluation and storage of biological specimens for genetic or molecular analysis in this trial/future use $\{33\}$

Page 14/30 


\section{Statistical methods}

\section{Statistical methods for primary and secondary outcomes $\{20 \mathrm{a}\}$}

We will conduct descriptive analyses, including examining means, proportions, and variability for each variable overall and by intervention arm. Then we will fit models to test the aims' hypotheses. Tests of statistical significance will be two-sided and a priori statistical significance will be set at $P<0.05$.

To determine whether GROWel/ was efficacious at reducing the proportion of women who have excess GWG we will create an indicator variable for excess weight gain during pregnancy using IOM guidelines. If a participant's prepregnancy BMI is in the overweight category $(25<30)$ and the difference between the weight at delivery and prepregnancy weight is greater than 25 pounds, then the indicator will be 1 . If a participant's prepregnancy BMI is in the obese class I $(30<35)$ or obese class II $(35-40)$ categories and the difference between the weight at delivery and prepregnancy weight is greater than 20 pounds, then the indicator will be 1. A series of logistic regression models will be fitted to estimate the association between excess weight gain during pregnancy and the arms (GROWell and attention control). The first model will control only for BMI groups, race/ethniciy, and recruitment source (e.g., clinic or community). The second model will include baseline demographic variables (age, parity, diet quality, physical activity). The last model will add the group arm (e.g., GROWell or attention control).

Next we will compare the efficacy of GROWell to the attention control in reducing postpartum weight retention at 6 months postpartum as measured by the proportion of women who are within $5 \%$ of their pre-pregnancy weight while controlling for demographics, parity, physical activity, diet quality, breastfeeding status, and depression/anxiety. Hypothesis: The proportion of women who retain pregnancy weight will be significantly lower for those in the GROWell arm than those in the attention control arm. An indicator variable for postpartum weight retention will take the value of 1 if a participant's 6-month postpartum weight is greater than 1.05 of the participant's prepregnancy weight, and 0 otherwise. A similar series of logistic regression models estimated in aim 1 will be fitted to estimate the association between postpartum weight retention and the intervention. Results from each model will be presented.

Exploratory Outcomes. Relative frequencies and crude adjusted odds ratios will be computed for the maternal, obstetric, and neonatal outcomes by intervention arm. Adjusted odds ratios will be computed for the outcomes by intervention arm to obtain information about the strength of the association of intervention on the outcome. Control variables will include demographics, diet quality, physical activity, and depression/anxiety.

\section{Interim analyses $\{21 \mathrm{~b}\}$}


The GROWell trial has a DSMB. The DSMB will receive data from the PI about the trial participants every 6 months and will determine if unblinding needs to occur to remove specific participants from the trial or terminate the trial.

This study will be stopped prior to its completion if any of the following conditions apply: (1) the intervention is associated with AEs that suggest the intervention is not safe; (2) difficulty in study recruitment or retention will significantly impact the ability to evaluate the study endpoints; (3) any new information becomes available during the trial that necessitates stopping the trial; or (4) other situations occur that might warrant stopping the trial.

\section{Methods for additional analyses (e.g. subgroup analyses) \{20b\}}

N/A

\section{Methods in analysis to handle protocol non-adherence and any statistical methods to handle missing data $\{20 \mathrm{c}\}$}

Our primary outcomes necessitate accurate weights; however, we will enroll women through 16 weeks gestation who may not have a first trimester weight. We will address discrepancies in measuring preconception weight and delivery weight by using propensity score matching. A propensity score is the likelihood of being treated and is expressed as a linear combination of all background variables. $(78,79)$ With propensity score matching, we can treat missing and non-missing values as treated and untreated subjects and the likelihood of missing as the propensity scores. The strategy of using propensity score matching to handle missing data on multiple variables is more effective than other approaches, because propensity score adjustment reduces imbalance on all the background variables at once. Using the imputed weights will ensure that variance in preconception and delivery weight estimates as a function of later entry into prenatal care, inaccurate recall, variance in gestational age at delivery, variance in clinic scales, and other sources of error is minimized.

Plans to give access to the full protocol, participant level-data and statistical code $\{31 \mathrm{c}\}$

N/A

Oversight and monitoring

Composition of the coordinating centre and trial steering committee $\{5 \mathrm{~d}\}$

$\mathrm{N} / \mathrm{A}$

Composition of the data monitoring committee, its role and reporting structure $\{21 \mathrm{a}\}$

A Data and Safety Monitoring Board (DSMB) has been established for this study. The DSMB will act in an advisory capacity to monitor participant safety, evaluate the progress of the study, and review procedures for maintaining the confidentiality of data, the quality of data collection, management, and 
analyses. None of the individuals serving on the DSMB are involved with this study, and each will sign a confidentiality and conflict of interest form. Monthly progress reports, including participant recruitment, retention/attrition, and AEs, will be provided to the DSMB chair. The advisory board will meet at least every 6 months to review study progress, including enrollment, retention, adherence, data quality, and any safety issues that may arise during the course of this study.

DSMB Charter. A DSMB charter will be established and signed by each member of the DSMB. The charter outlines responsibilities of the DSMB and its members. These include:

- Review the research protocol, informed consent documents, and plans for data safety and monitoring prior to subject enrollment;

- Recommend subject recruitment be initiated after receipt of a satisfactory protocol;

- Evaluate the progress of the trial, including periodic assessments of data quality and timeliness, recruitment, accrual and retention, participant risk versus benefit, performance of the trial sites, and other factors that can affect study outcome;

- Consider factors external to the study when relevant information becomes available, such as scientific or therapeutic developments that may have an impact on the safety of the participants or the ethics of the trial;

- Review study performance, make recommendations and assist in the resolution of problems reported by the Principal Investigator;

- Protect the safety of the study participants;

- Report to NINR on the safety and progress of the trial;

- Make recommendations to the NINR and the Principal Investigator concerning continuation, termination, or other modifications of the trial based on the observed beneficial or adverse effects of the treatment under study;

- If appropriate, review interim analyses in accordance with stopping rules, which are clearly defined in advance of data analysis and have the approval of the DSMB;

- Ensure the confidentiality of the study data and the results of monitoring;

- Assist the NINR by commenting on any problems with study conduct, enrollment, sample size, and/or data collection.

\section{Adverse event reporting and harms $\{22\}$}

We will monitor pregnancy-related AEs/SAEs at mid and late pregnancy during scheduled online data collection assessments, as well as when we collect labor and delivery data. We also will ask women to inform us of any events between data collection time points. Women will be discontinued from the intervention if they experience a pregnancy-related event and events will be documented and categorized for our exploratory aim. 
AEs will be labeled according to severity, which is based on their impact on the participant. An AE will be termed "mild" if it does not have a major impact, "moderate" if it causes the participant some minor inconvenience, and "severe" if it causes a substantial disruption to the participant's well-being.

Additionally, all AEs/SAEs will be categorized according to the likelihood that they are related to the study intervention (definitely unrelated, definitely related, probably related, or possibly related).

SAEs that are unanticipated, serious, and possibly related to the study intervention will be reported to the DSMB, IRB, and NINR in accordance with requirements. Unexpected fatal or life-threatening AEs related to the intervention will be reported to the NINR Program Officer within 7 days. Other serious and unexpected AEs related to the intervention will be reported to the NINR Program Official within 15 days. Anticipated or unrelated SAEs will be handled in a less urgent manner but will be reported to the DSMB, IRB, NINR, and other oversight organizations in accordance with their requirements. In the annual AE summary, the DSMB report will state that they have reviewed all AE reports.

\section{Frequency and plans for auditing trial conduct $\{23\}$}

Monthly audits will be performed to ensure recruitment milestones are being met and to identify and correct any potential bias in methods and/or data. These audits will be performed by the study statistician and PI.

\section{Plans for communicating important protocol amendments to relevant parties (e.g. trial participants, ethical committees) $\{25\}$}

If we determine that protocol modifications are necessary, we will inform the UC Davis IRB, our DSMB, the $\mathrm{NIH} \mathrm{NINR,} \mathrm{and} \mathrm{update} \mathrm{our} \mathrm{trial} \mathrm{registration} \mathrm{at} \mathrm{ClinicalTrials.gov.}$

\section{Dissemination plans $\{31$ a $\}$}

We will communicate findings in peer-reviewed presentations and publications, on UC Davis websites, at UC Davis sponsored grand rounds, and to participants.

GROWel/ is registered with ClinicalTrials.gov and is funded by the NIH and thus will follow NIH Guide Notice NOT-OD-16-149 to submit summary results to ClinicalTrials.gov for public posting. Registration information includes descriptive information, recruitment information, location and contact information, and administrative data. Results from the trial will be submitted no later than one year following the trial's primary completion date. Results information will include participant flow, demographic and baseline characteristics, outcomes and statistical analyses, adverse events, the protocol and statistical analysis plan, and administrative information.

\section{Discussion}


Excess GWG and PPWR have significant negative long-term impacts on maternal and offspring health, including increased risk for poor pregnancy and birth outcomes and cardiometabolic disease across the lifespan for both mothers and their children. Thus, pregnancy and early postpartum/infancy are critical time periods for dietary interventions that may improve maternal-child health. We propose a large, RCT of a digital dietary intervention that addresses several key deficits in previous approaches to reducing GWG and associated postpartum weight retention. First, most studies that found no reductions in GWG provided generalized dietary education rather than content specific to pregnancy or the participant's needs and goals.(30) Indeed, previous reviews of dietary interventions in pregnancy have noted that future interventions should include specific, person-centered dietary goals to improve success rates,(30, 80) which GROWell offers. Second, among the studies that did find positive intervention effects on GWG, the majority were high intensity and involved either in-person or telephonic educational and counseling sessions, some lasting 1.5 to 2 hours over multiple visits. $(29,30)$ While the literature supports the need for high-dose interventions to achieve positive behavioral change,(81) such approaches frequently require significant human resources to deliver these interventions, which limits generalizability in real world settings. GROWell offers high-intensity support using digital health, thereby providing the level of contact necessary for change without the need for significant human resources. Third, few technology-based interventions have been investigated in large-scale RCTs.(42) This is a significant missed opportunity given that among women ages 18-44 across all race/ethnicities, incomes, educational levels, and geographic locations, at least $90 \%$ own cell phones and at least $72 \%$ own smart phones.(82) Young adult women have high penetration of eHealth use, or using mobile and smart phones, Internet, and social media for general health information seeking and sharing,(83) and pregnancy-specific information, including gestational weight gain.(84) In fact, national data show that being young and female is a consistent predictor of eHealth use more than any other demographic.(85) Thus, we are using an approach that is likely to resonate with women of childbearing age across all socioeconomic statuses, geographic locations, and race/ethnicities. Lastly, and importantly, no previous interventions have extended into the postpartum period, but rather, ended in the late third trimester despite calls for interpregnancy weight control strategies(86-89) and strong evidence that postpartum weight retention six months post-birth is highly prevalent and increases women's risk for poor midlife health, including morbid obesity, related cardiometabolic disease, and cancer.(18) Continuing GROWel/ through 6-months postpartum may provide women with longer-term support that has the capacity to improve dietary intake well beyond pregnancy, including supporting interpartum health for women who have future children.

During the current COVID-19 pandemic, conducting non-essential clinical trials has become ever more challenging. However, there is also a unique opportunity to use technology to allow access to clinical trials to previously underrepresented participants.(90) Before the pandemic, we included in-person study visits that would have been difficult for us to accomplish for our more rural participants or for those with transportation, time, or other barriers. Now we are able to reach women at any place in our catchment area, which spans from Placer County to Marin County and south to Santa Clara County in CA. Additionally, women who may have not wanted to participate due to the in-person requirement can now participate entirely remotely. Making a clinical trial completely virtual is not feasible for all studies, but it 
worked well for GROWell after a few modifications, including creating options for: (1) the ability to provide a signed consent via DocuSign, (2) mailing a Bluetooth scale for weight data that interfaces with our application, and (3) online surveys rather than in-person data collection.

Novel approaches to maintaining GWG within IOM-recommended ranges and supporting women to lose pregnancy weight are critical to advancing women's health across the lifespan. Additionally, given the rise in children's rates of overweight and obesity and associated cardiometabolic conditions (e.g., hypertension, hypercholesterolemia) that have been linked to intrauterine exposures (e.g., maternal diet, overweight/obesity, and adiposity) and dietary consumption in early infancy, prenatal and early postpartum interventions are likely to have the greatest impact on lifelong health. Establishing GROWell as effective at reducing GWG and postpartum weight retention can provide a foundation for using digital health to promote healthy behaviors during pregnancy for optimal maternal-child health.

\section{Trial Status}

Currently, this project (protocol version 1, October 15, 2020) is in pre-enrollment. We are finalizing platform delivery and messaging, as well as developing recruitment materials in preparation for a December 1, 2020 start-date for recruitment. Preliminary results on recruitment success, including baseline demographics of the sample are expected in late 2022. Outcomes associated with GWG and pregnancy are expected in late 2023, while outcomes on postpartum weight retention GROWell adherence are expected in late 2024.

\section{Abbreviations}

GROWell: Goals for Reaching Optimal Wellness; GWG: gestational weight gain; IOM: Institute of Medicine; BMI: body mass index; PPWR: postpartum weight retention; RCT: randomized controlled trial; BMI: body mass index; CRC: clinical research coordinator; IRB: institutional review board; DSMB: data safety monitoring board; NIH NINR: National Institutes of Health National Institute for Nursing Research; EHR: electronic health record; NICU: neonatal intensive care unit; DHQ-II: Diet History Questionnaire-II; KPAS: Kaiser Physical Activity Survey; ASA24®: Automated Self-Administered 24-Hour Dietary Assessment Tool; AHEI-2015: Alternate Healthy Eating Index-2015; EPDS: Edinburgh Postnatal Depression Scale; SAR: selfmonitoring adherence rates; GAR: goal adherence rates

\section{Declarations}

\section{Acknowledgements}

The authors acknowledge Dori Steinberg, PhD, RD, Duke Global Digital Health, and Pattern Health for their assistance with programming and algorithmic personalization.

\section{Authors' contributions $\{31 \mathrm{~b}\}$}


LAS designed the study and drafted the protocol. JEP edited and revised the manuscript and contributed to the protocol development. CO, DN, and EB contributed to study design. SL contributed to the statistical analysis plan. $\mathrm{CO}$ and $\mathrm{CW}$ contributed to recruitment processes. $\mathrm{CW}$ was responsible for determining the clinical safety of the study design. All authors have read and approved the final manuscript.

\section{Funding $\{4\}$}

This study is funded by the National Institutes of Health, National Institute of Nursing Research, R01NR017659.

\section{Availability of data and materials $\{29\}$}

Not applicable.

\section{Ethics approval and consent to participate $\{24\}$}

The University of California, Davis and Duke University IRBs have approved this protocol and informed consent will be required by all participants prior to participation. A data safety monitoring committee is in place for this study.

\section{Consent for publication $\{32\}$}

Consent for publication is not applicable because this article does not contain any data.

\section{Competing interests $\{28\}$}

The authors declare no competing interests.

\section{References}

1. Braun PR, Han S, Hing B, Nagahama Y, Gaul LN, Heinzman JT, et al. Genome-wide DNA methylation comparison between live human brain and peripheral tissues within individuals. Transl Psychiatry. 2019;9(1):47.

2. Deputy NP, Sharma AJ, Kim SY, Hinkle SN. Prevalence and characteristics associated with gestational weight gain adequacy. Obstet Gynecol. 2015;125(4):773-81.

3. Baeten JM, Bukusi EA, Lambe M. Pregnancy complications and outcomes among overweight and obese nulliparous women. Am J Public Health. 2001;91(3):436-40.

4. Cedergren M. Effects of gestational weight gain and body mass index on obstetric outcome in Sweden. Int J Gynecol Obstet2006. p. 269-74.

5. Crane JMG, White J, Murphy P, Burrage L, Hutchens D. The Effect of Gestational Weight Gain by Body Mass Index on Maternal and Neonatal Outcomes. Journal of Obstetrics and Gynaecology 
Canada2009.

6. Dietl J. Maternal obesity and complications during pregnancy. J Perinat Med. 2005;33(2):100-5.

7. O'Brien TE, Ray JG, Chan WS. Maternal body mass index and the risk of preeclampsia: a systematic overview. Epidemiology. 2003;14(3):368-74.

8. Oken E, Rifas-Shiman SL, Field AE, Frazier AL, Gillman MW. Maternal gestational weight gain and offspring weight in adolescence. Obstet Gynecol. 2008;112(5):999-1006.

9. Oken E, Taveras EM, Kleinman KP, Rich-Edwards JW, Gillman MW. Gestational weight gain and child adiposity at age 3 years. American Journal of Obstetrics and Gynecology2007.

10. Olson CM, Strawderman MS, Hinton PS, Pearson TA. Gestational weight gain and postpartum behaviors associated with weight change from early pregnancy to 1 y postpartum. Int J Obes Relat Metab Disord. 2003;27(1):117-27.

11. Schack-Nielsen L, Michaelsen KF, Gamborg M, Mortensen EL, Sørensen TIA. Gestational weight gain in relation to offspring body mass index and obesity from infancy through adulthood. International Journal of Obesity2010.

12. Scholl TO, Hediger ML, Schall JI, Ances IG, Smith WK. Gestational weight gain, pregnancy outcome, and postpartum weight retention. Obstet Gynecol. 1995;86(3):423-7.

13. Soltani H, Fraser RB. A longitudinal study of maternal anthropometric changes in normal weight, overweight and obese women during pregnancy and postpartum. $\mathrm{Br} J$ Nutr. 2000;84(1):95-101.

14. Weiss JL, Malone FD, Emig D, Ball RH, Nyberg DA, Comstock $\mathrm{CH}$, et al. Obesity, obstetric complications and cesarean delivery rate--a population-based screening study. Am J Obstet Gynecol. 2004;190(4):1091-7.

15. Kac G, Benicio MH, Velásquez-Meléndez G, Valente JG. Nine months postpartum weight retention predictors for Brazilian women. Public Health Nutrition. 2004.

16. Linné Y, Dye L, Barkeling B, Rössner S. Weight development over time in parous women - The SPAWN study: 15 Years follow-up. International Journal of Obesity. 2003.

17. Soltani H, Fraser RB. A longitudinal study of maternal anthropometric changes in normal weight, overweight and obese women during pregnancy and postpartum. British Journal of Nutrition. 2000.

18. The Role of Postpartum Weight Retention in Obesity Among Women: A Review of the Evidence, (2003).

19. Gaillard R, Durmuş B, Hofman A, MacKenbach JP, Steegers EAP, Jaddoe VWV. Risk factors and outcomes of maternal obesity and excessive weight gain during pregnancy. Obesity. 2013. 
20. Nehring I, Schmoll S, Beyerlein A, Hauner H, Von Kries R. Gestational weight gain and long-term postpartum weight retention: A meta-analysis. American Journal of Clinical Nutrition. 2011.

21. Mannan M, Doi SA, Mamun AA. Association between weight gain during pregnancy and postpartum weight retention and obesity: A bias-adjusted meta-analysis. Nutrition Reviews2013.

22. Hochner H, Friedlander Y, Calderon-Margalit R, Meiner V, Sagy Y, Avgil-Tsadok M, et al. Associations of maternal prepregnancy body mass index and gestational weight gain with adult offspring cardiometabolic risk factors: The jerusalem perinatal family follow-up study. Circulation2012.

23. Kaar JL, Crume T, Brinton JT, Bischoff KJ, McDuffie R, Dabelea D. Maternal obesity, gestational weight gain, and offspring adiposity: The exploring perinatal outcomes among children study. Journal of Pediatrics2014.

24. Mamun AA, Mannan M, Doi SAR. Gestational weight gain in relation to offspring obesity over the life course: A systematic review and bias-adjusted meta-analysis. Obesity Reviews2014.

25. Gestational weight gain, prepregnancy body mass index and offspring attention-deficit hyperactivity disorder symptoms and behaviour at age 10, (2016).

26. Pugh SJ, Richardson GA, Hutcheon JA, Himes KP, Brooks MM, Day NL, et al. Maternal Obesity and Excessive Gestational Weight Gain Are Associated with Components of Child Cognition. The Journal of Nutrition2015.

27. Kominiarek MA, Peaceman AM. Gestational weight gain. Am J Obstet Gynecol. 2017;217(6):64251.

28. Deputy NP, Sharma AJ, Kim SY. Gestational Weight Gain - United States, 2012 and 2013. MMWR Morb Mortal Wkly Rep. 2015;64(43):1215-20.

29. Quinlivan JA, Julania S, Lam L. Antenatal dietary interventions in obese pregnant women to restrict gestational weight gain to Institute of Medicine recommendations: a meta-analysis. Obstet Gynecol. 2011;118(6):1395-401.

30. Flynn AC, Dalrymple K, Barr S, Poston L, Goff LM, Rogozińska E, et al. Dietary interventions in overweight and obese pregnant women: A systematic review of the content, delivery, and outcomes of randomized controlled trials. Nutrition Reviews2016. p. 312-28.

31. Harrison CL, Lombard CB, Strauss BJ, Teede HJ. Optimizing healthy gestational weight gain in women at high risk of gestational diabetes: a randomized controlled trial. Obesity (Silver Spring). 2013;21(5):904-9.

32. Vinter CA, Jensen DM, Ovesen P, Beck-Nielsen H, Jorgensen JS. The LiP (Lifestyle in Pregnancy) study: a randomized controlled trial of lifestyle intervention in 360 obese pregnant women. Diabetes Care. 
33. Quinlivan JA, Lam LT, Fisher J. A randomised trial of a four-step multidisciplinary approach to the antenatal care of obese pregnant women. Aust N Z J Obstet Gynaecol. 2011;51(2):141-6.

34. Guelinckx I, Devlieger R, Mullie P, Vansant G. Effect of lifestyle intervention on dietary habits, physical activity, and gestational weight gain in obese pregnant women: a randomized controlled trial. Am J Clin Nutr. 2010;91(2):373-80.

35. Vesco KK, Karanja N, King JC, Gillman MW, Leo MC, Perrin N, et al. Efficacy of a group-based dietary intervention for limiting gestational weight gain among obese women: a randomized trial. Obesity (Silver Spring). 2014;22(9):1989-96.

36. Poston L, Bell R, Croker H, Flynn AC, Godfrey KM, Goff L, et al. Effect of a behavioural intervention in obese pregnant women (the UPBEAT study): A multicentre, randomised controlled trial. The Lancet Diabetes and Endocrinology2015.

37. Web-based interventions for behavior change and self-management: Potential, pitfalls, and progress, (2012).

38. Simmons LA. Goals for reaching optimal wellness: GROWell. Duke University School of Nursing Center for Nursing Research Pilot Grant. 2016.

39. Soltani H, Duxbury AMS, Arden MA, Dearden A, Furness PJ, Garland C. Maternal obesity management using mobile technology: A feasibility study to evaluate a text messaging based complex intervention during pregnancy. Journal of Obesity2015.

40. Graham ML, Uesugi KH, Niederdeppe J, Gay GK, Olson CM. The theory, development, and implementation of an e-intervention to prevent excessive gestational weight gain: E-Moms Roc. Telemedicine and e-Health2014.

41. Jackson RA, Stotland NE, Caughey AB, Gerbert B. Improving diet and exercise in pregnancy with Video Doctor counseling: A randomized trial. Patient Education and Counseling2011.

42. O'Brien OA, McCarthy M, Gibney ER, McAuliffe FM. Technology-supported dietary and lifestyle interventions in healthy pregnant women: A systematic review. European Journal of Clinical Nutrition2014.

43. Bandura A. Social Cognitive Theory of Self-Regulation. Organ Behav Hum Dec. 1991;50(2):24887.

44. Hall PA, Fong GT. Temporal self-regulation theory: A model for individual health behavior. Health Psychology Review. 2007;1(1):6-52. 
45. Schnoll R, Zimmerman BJ. Self-regulation training enhances dietary self-efficacy and dietary fiber consumption. J Am Diet Assoc. 2001;101(9):1006-11.

46. Ryan RM, Deci EL. Self-regulation and the problem of human autonomy: Does psychology need choice, self-determination, and will? J Pers. 2006;74(6):1557-85.

47. Implicit processes, self-regulation, and interventions for behavior change, (2017).

48. Dildy GA, Jackson GM, Fowers GK, Oshiro BT, Varner MW, Clark SL. Very advanced maternal age: Pregnancy after age 45. American Journal of Obstetrics and Gynecology 1996.

49. Luke B, Brown MB. Elevated risks of pregnancy complications and adverse outcomes with increasing maternal age. Human Reproduction2007.

50. Cnattingius S, Lambe M. Trends in smoking and overweight during pregnancy: Prevalence, risks of pregnancy complications, and adverse pregnancy outcomes. Seminars in Perinatology2002.

51. Hammoud AO, Bujold E, Sorokin Y, Schild C, Krapp M, Baumann P, et al. Smoking in pregnancy revisited: Findings from a large population-based study 2005.

52. Aubin HJ, Farley A, Lycett D, Lahmek P, Aveyard P. Weight gain in smokers after quitting cigarettes: Meta-analysis. BMJ (Online)2012.

53. Ferrara CM, Kumar M, Nicklas B, McCrone S, Goldberg AP. Weight gain and adipose tissue metabolism after smoking cessation in women. International Journal of Obesity 2001.

54. Smoking cessation and weight gain, (2004).

55. Nestler EJ, Barrot M, DiLeone RJ. Neurobiology of depression. Neuron. European Journal of Pharmacology2002.

56. Ouwens MA, van Strien T, van Leeuwe JFJ. Possible pathways between depression, emotional and external eating. A structural equation model. Appetite2009.

57. Demanding attention: Reconsidering the role of attention control groups in behavioral intervention research, (2013).

58. Pagoto SL, McDermott MM, Reed G, Greenland P, Mazor KM, Ockene JK, et al. Can attention control conditions have detrimental effects on behavioral medicine randomized trials? Psychosomatic Medicine2013.

59. Attention placebo control in randomized controlled trials of psychosocial interventions: Theory and practice, (2015). 
60. Bennett GG, Foley P, Levine E, Whiteley J, Askew S, Steinberg DM, et al. Behavioral treatment for weight gain prevention among black women in primary care practice: A randomized clinical trial. JAMA Internal Medicine2013.

61. Steinberg DM, Levine EL, Lane I, Askew S, Foley PB, Puleo E, et al. Adherence to self-monitoring via interactive voice response technology in an ehealth intervention targeting weight gain prevention among black women: Randomized controlled trial. Journal of Medical Internet Research2014.

62. ACOG Committee Opinion No. 548: Weight Gain During Pregnancy2013.

63. Flick AA, Brookfield KF, De La Torre L, Tudela CM, Duthely L, González-Quintero VH. Excessive weight gain among obese women and pregnancy outcomes. American Journal of Perinatology 2010.

64. Alberico S, Montico M, Barresi V, Monasta L, Businelli C, Soini V, et al. The role of gestational diabetes, pre-pregnancy body mass index and gestational weight gain on the risk of newborn macrosomia: results from a prospective multicentre study. Bmc Pregnancy Childb. 2014;14.

65. Subar AF, Kirkpatrick SI, Mittl B, Zimmerman TP, Thompson FE, Bingley C, et al. The Automated Self-Administered 24-Hour Dietary Recall (ASA24): A Resource for Researchers, Clinicians, and Educators from the National Cancer Institute. J Acad Nutr Diet. 2012;112(8):1134-7.

66. Guenther PM, Kirkpatrick SI, Reedy J, Krebs-Smith SM, Buckman DW, Dodd KW, et al. The Healthy Eating Index-2010 Is a Valid and Reliable Measure of Diet Quality According to the 2010 Dietary Guidelines for Americans. The Journal of Nutrition2014.

67. Miller PE, Mitchell DC, Harala PL, Pettit JM, Smiciklas-Wright H, Hartman TJ. Development and evaluation of a method for calculating the Healthy Eating Index-2005 using the Nutrition Data System for Research. Public Health Nutrition2011.

68. Rifas-Shiman SL, Rich-Edwards JW, Kleinman KP, Oken E, Gillman MW. Dietary Quality during Pregnancy Varies by Maternal Characteristics in Project Viva: A US Cohort. J Am Diet Assoc2009.

69. Ainsworth BE, Sternfeld B, Richardson MT, Jackson K. Evaluation of the Kaiser Physical Activity Survey in women. Medicine and Science in Sports and Exercise2000.

70. Schmidt MD, Freedson PS, Pekow P, Roberts D, Sternfeld B, Chasan-Taber L. Validation of the Kaiser Physical Activity Survey in pregnant women. Medicine and Science in Sports and Exercise2006.

71. Cox JL, Holden JM, Sagovsky R. Detection of Postnatal Depression: Development of the 10-item Edinburgh Postnatal Depression scale. British Journal of Psychiatry1987.

72. Review of validation studies of the Edinburgh postnatal depression scale, (2001). 
73. Matthey S, Henshaw C, Elliott S, Barnett B. Variability in use of cut-off scores and formats on the Edinburgh Postnatal Depression Scale - Implications for clinical and research practice. Archives of Women's Mental Health2006.

74. Matthey S. Using the Edinburgh Postnatal Depression Scale to screen for anxiety disorders. Depression and anxiety2008.

75. Simmons LA, editor Beyond the Genome: Advancing Precision Medicine through Personalized Health Behavior Change Interventions. American Academy of Health Behavior Annual Scientific Meeting; 2017; Tucson, AZ.

76. Herring SJ, Cruice JF, Bennett GG, Rose MZ, Davey A, Foster GD. Preventing excessive gestational weight gain among African American women: A randomized clinical trial. Obesity2016.

77. Yang NY, Wroth S, Parham C, Strait M, Simmons LA. Personalized Health Planning with Integrative Health Coaching to Reduce Obesity Risk among Women Gaining Excess Weight during Pregnancy. Global Advances in Health and Medicine2013.

78. Pan W, Bai H. Propensity score interval matching: using bootstrap confidence intervals for accommodating estimation errors of propensity scores. BMC Medical Research Methodology. 2015;15(1):53.

79. Pan W, Bai H. Propensity Score Analysis: Fundamentals and Developments: Guilford Publications; 2015.

80. Olander EK, Berg M, McCourt C, Carlström E, Dencker A. Person-centred care in interventions to limit weight gain in pregnant women with obesity - a systematic review. Bmc Pregnancy Childb2015.

81. Greaves CJ, Sheppard KE, Abraham C, Hardeman W, Roden M, Evans PH, et al. Systematic review of reviews of intervention components associated with increased effectiveness in dietary and physical activity interventions. Bmc Public Health. 2011;11.

82. Demographics of Mobile Device Ownership and Adoption in the United States | Pew Research Center.

83. U.S. Technology Device Ownership 2015 | Pew Research Center.

84. Willcox JC, Campbell KJ, McCarthy EA, Lappas M, Ball K, Crawford D, et al. Gestational weight gain information: Seeking and sources among pregnant women. Bmc Pregnancy Childb2015.

85. Kontos E, Blake KD, Chou WYS, Prestin A. Predictors of ehealth usage: Insights on the digital divide from the health information national trends survey 2012. Journal of Medical Internet Research2014. 
86. ACOG Committee opinion no. 549: Obesity in Pregnancy. Obstetrics and gynecology. 2013;121(1):213-7.

87. Bogaerts A, Van Den Bergh BRH, Ameye L, Witters I, Martens E, Timmerman D, et al. Interpregnancy weight change and risk for adverse perinatal outcome2013.

88. Jain AP, Gavard JA, Rice JJ, Catanzaro RB, Artal R, Hopkins SA. The impact of interpregnancy weight change on birthweight in obese women. American Journal of Obstetrics and Gynecology2013.

89. Villamor E, Cnattingius S. Interpregnancy weight change and risk of adverse pregnancy outcomes: a population-based study. Lancet2006.

90. Noonan D, Simmons LA. Navigating Nonessential Research Trials During COVID19: The Push We Needed For Using Digital Technology To Increase Access For Rural Participants? J Rural Health. 2020.

\section{Figures}

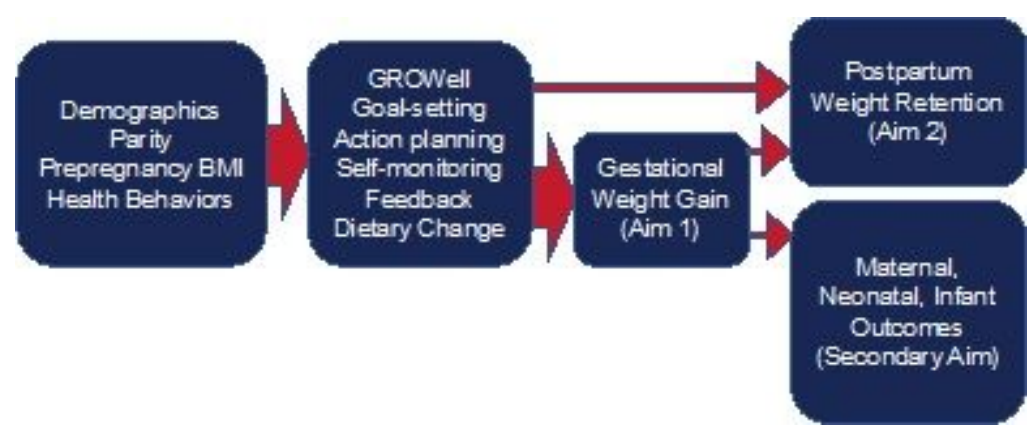

\section{Figure 1}

Conceptual model for GROWell
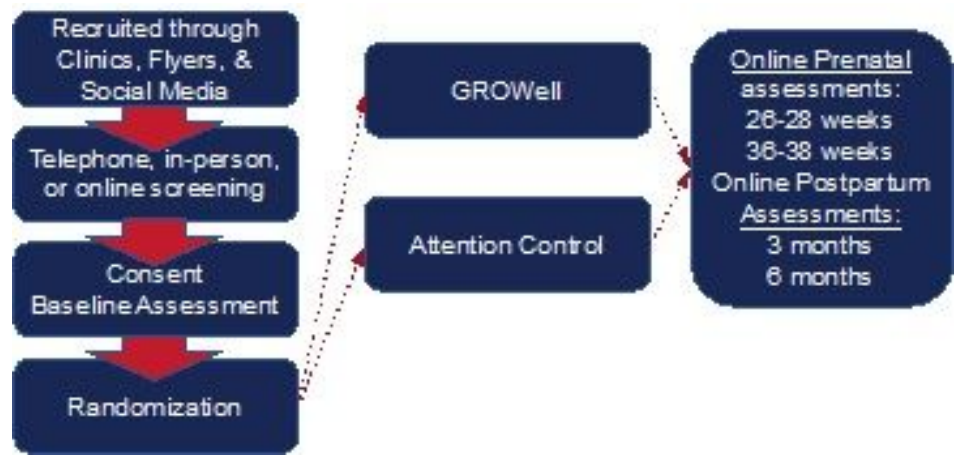

\section{Figure 2}

Overview of study design 

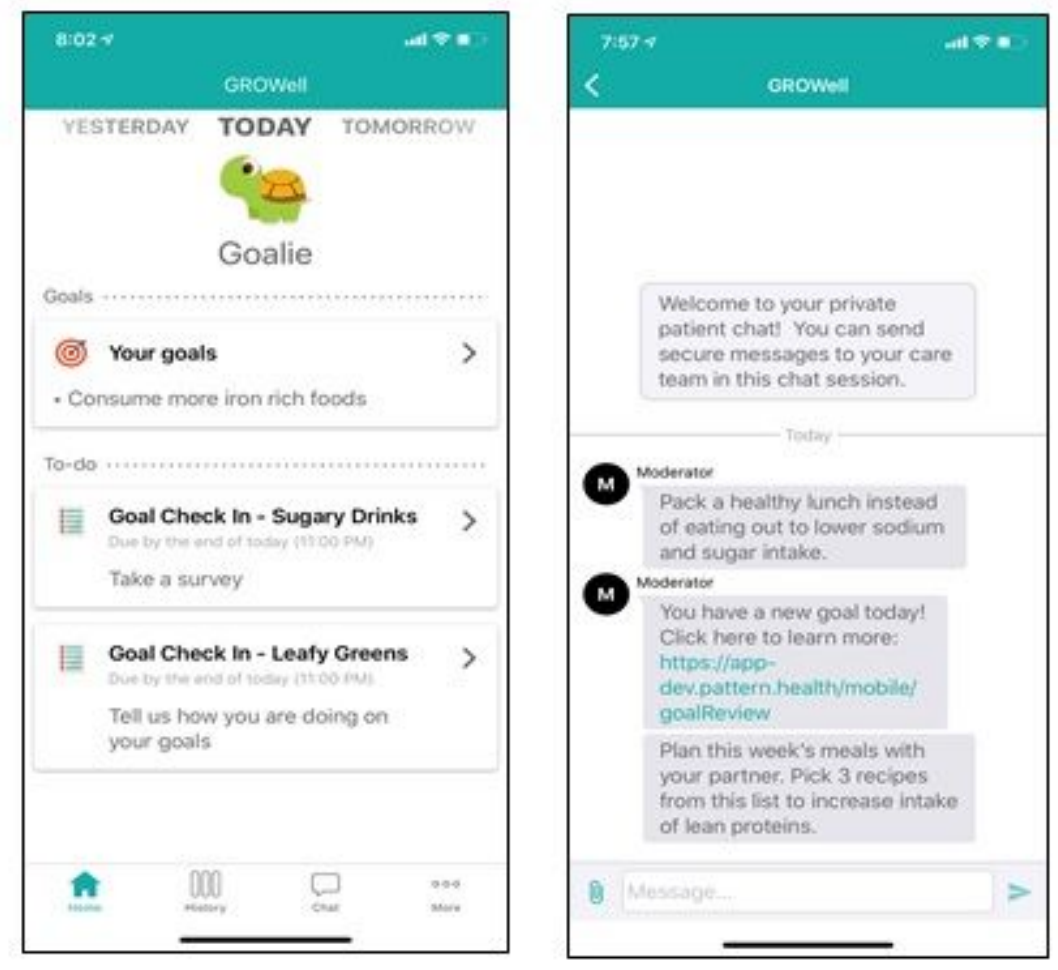

Figure 3

Example view of the mHealth platform and text messages 


\begin{tabular}{|c|c|c|c|c|c|c|}
\hline \multirow[b]{3}{*}{ TIMEPOINT $^{\star \star *}$} & \multicolumn{6}{|c|}{ STUDY PERIOD } \\
\hline & \multirow{2}{*}{$\frac{\text { Enrollment }}{-t_{1}}$} & \multirow{2}{*}{$\frac{\text { Allocation }}{0}$} & \multicolumn{2}{|c|}{ Prenatal } & \multicolumn{2}{|c|}{ Postpartum } \\
\hline & & & $t_{1}$ & $t_{2}$ & $t_{3}$ & $t_{4}$ \\
\hline \multicolumn{7}{|l|}{ ENROLLMENT: } \\
\hline \multirow{2}{*}{$\begin{array}{l}\text { Eligibility screen } \\
\text { Informed consent }\end{array}$} & $X$ & & & & & \\
\hline & $X$ & & & & & \\
\hline Allocation & & $X$ & & & & \\
\hline \multicolumn{7}{|l|}{ INTERVENTIONS: } \\
\hline \multicolumn{7}{|l|}{ GROWell } \\
\hline \multicolumn{7}{|l|}{ Attention Control } \\
\hline \multicolumn{7}{|l|}{ ASSESSMENTS: } \\
\hline \multirow{2}{*}{$\begin{array}{r}\text { Baseline } \\
\text { Assessment } \\
26-28 \text { week } \\
\text { assessment }\end{array}$} & $X$ & & & & & \\
\hline & & & $\mathrm{X}$ & & & \\
\hline \multirow{3}{*}{$\begin{array}{r}36-38 \text { week } \\
\text { assessment } \\
3 \text { months } \\
\text { postpartum } \\
\text { assessment } \\
6 \text { months } \\
\text { postpartum } \\
\text { assessment }\end{array}$} & & & & $\mathrm{X}$ & & \\
\hline & & & & & $X$ & \\
\hline & & & & & & $\mathrm{X}$ \\
\hline
\end{tabular}

Figure 4

Schedule of enrollment, interventions, and assessments. 